INPLASY

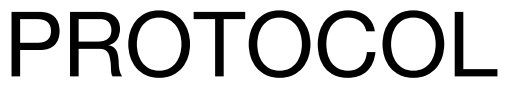

To cite: Baskoro et al. Hypnotic Based Intervention for people with Non-Communicable Diseases : A scoping review. Inplasy protocol 202130040. doi:

10.37766/inplasy2021.3.0040

Received: 13 March 2021

Published: 13 March 2021

Corresponding author:

Danang Baskoro

danangsetyobudibaskoro@gmail.com

Author Affiliation:

Gajah Mada University

Support: None declared.

Review Stage at time of this submission: The review has not yet started.

Conflicts of interest:

None declared.

\section{Hypnotic Based Intervention for people with Non-Communicable Diseases : A scoping review}

\author{
Baskoro, D1; Gandawijaya, W2; Yuniarti, K³.
}

Review question / Objective: To describe the research question, the researcher used the PICOS framework. The purpose of conducting this scoping review is to systematically map the research literature that can be accessed to answer research questions. The four specific research questions posed are as follows: What types of Hypnosis-Based Interventions are used for people with Non-Communicable Diseases? What Types of Non-Communicable Diseases using Hypnosis-Based Interventions? What are the results that were researched to be targeted in Hypnotic Based Therapy research in people with non-communicable diseases? Is there evidence of the effectiveness of Hypnotic Based Interventions? This study will examine research using quantitative and qualitative methods. Through this process the answers to the questions found will produce a clearer picture of the effectiveness of Hypnotic Based Interventions in Patients with Non-Communicable Diseases. The long-term objective of this study is to provide views for sufferers, families and health workers about psychological therapy to help optimal treatment and improve psychological well-being.

INPLASY registration number: This protocol was registered with the International Platform of Registered Systematic Review and Meta-Analysis Protocols (INPLASY) on 13 March 2021 and was last updated on 13 March 2021 (registration number INPLASY202130040).

\section{INTRODUCTION}

Review question / Objective: To describe the research question, the researcher used the PICOS framework. The purpose of conducting this scoping review is to systematically map the research literature that can be accessed to answer research questions. The four specific research questions posed are as follows: What types of Hypnosis-Based Interventions are used for people with Non-Communicable Diseases? What Types of NonCommunicable Diseases using Hypnosis- 
Based Interventions? What are the results that were researched to be targeted in Hypnotic Based Therapy research in people with non-communicable diseases? Is there evidence of the effectiveness of Hypnotic Based Interventions? This study will examine research using quantitative and qualitative methods. Through this process the answers to the questions found will produce a clearer picture of the effectiveness of Hypnotic Based Interventions in Patients with NonCommunicable Diseases. The long-term objective of this study is to provide views for sufferers, families and health workers about psychological therapy to help optimal treatment and improve psychological well-being.

Rationale: Non Communicable Diseases kills at least $\mathbf{4 1}$ million people every year. Although detection, screening and treatment, as well as palliative care, are essential for the management of NCDs, psychosocial interventions also need attention. This is important because many of the people with NCDs have psychological issues. In addition, having to take medication for life can also make patients feel bored, bored and ultimately refuse to continue treatment. The behavior of patients who support the treatment of their disease is also quite influenced by their psychological condition. Therefore, if psychosocial problems can be resolved, it is hoped that the medical treatment process will be more optimal. Yapko (2015) states that $\mathrm{HGI}$ is a type of Clinical Hypnosis approach to dealing with diseases and various mental conditions. Meanwhile, the mental state of hypnosis itself is a brain condition that has alpha or tetha dominant waves. Hypnotic awareness is characterized by focused attention, reduced ability to critique information and easy acceptance of suggestions. Many studies have examined the effectiveness of HGI for reducing negative emotions and creating / strengthening expectations. As noted by Wichowski \& Jubsch (1999), patient self-care is improved by listening to Guided Imagery recordings. Likewise, negative emotions such as depression and anxiety can be reduced through relaxation and Self Hypnosis Guided Imagery (Davidson, Fambach \& Richardson, 1978; Stetter, Walter et al, 1994).

Condition being studied: Several studies have revealed the use of Hypnotic Guided Imagery in people with Non Communicable Diseases. However, because of the many types of Non Communicable Diseases and the variety of outcomes studied by many studies, it is necessary to review several things related to the research questions posed by researchers. The questions are: What types of Hypnotic Based Interventions are used for people with Non Communicable Diseases? What types of Non Communicable Diseases use Hypnotic Based Interventions? What are the outcomes studied to be targeted in the Hypnotic Based Therapy study in patients with Non Communicable Diseases? Is there evidence of the effectiveness of Hypnotic Based Interventions?

\section{METHODS}

Search strategy: The search strategy will be carried out through the Cochrane Library, Google Schoolar, Scopus, Pubmed. The search will start on March 25, 2020 to April 25, 2020. The search will only be carried out on research that uses English and has been published in the last 20 years, namely 2001 to 2021 . The search will be carried out right before the final analysis and further studies taken for inclusion.

Participant or population: The study participants were people with noncommunicable diseases, such as cardiovascular disease (such as heart attacks and strokes), cancer, chronic respiratory diseases (such as chronic obstructive pulmonary disease and asthma), and diabetes. Female and male participants, at least 18 years old. Participants are still allowed to undergo the psychological therapy process. We excluded persons suffering from NonCommunicable Diseases with severe conditions, experiencing major clinical psychological disorders such as major depression and schizophrenia as well as 
organic disorders such as Alzheimer's and Epilepsy.

Intervention: This study will examine the types of Hypnotic Based Intervention used in patients with Non-Communicable Diseases, the types of Non-Communicable Diseases and the outcomes that have been studied and the evidence that shows the effectiveness of Hypnotic Based Intervention in Non-Communicable Diseases participants.

Comparator: The journal relevant to this study is a journal that describes the effectiveness of Hypnotic Based Interventions in people with NonCommunicable Diseases. Hypnotic Based Interventions that are input include hypnosis procedures with direct handling, procedures by listening to sound recordings, and other techniques that use the involvement of hypnosis awareness and suggestion.

Study designs to be included: We chose original articles, English and used primary data. The included studies used the Randomized Controlled Trial Approach. Researching the use of Hypnotic Based Interventions to patients with NonCommunicable Diseases.

Eligibility criteria: The journals that will be used are checked and matched with the inclusion criteria. The inclusion criteria chosen were journals on Hypnotic Based Interventions for patients with NonCommunicable Diseases. Female and male participants, at least 18 years old and still allow to undergo the psychological therapy process. While the approach used is the Rondomized Controlled Trial.

Information sources: Journals will be retrieved from various databases, namely the Cochrane Library, ProQuest, Scopus and Pubmed. If necessary, researchers will also search for data from gray literature through google schoolar.

Main outcome(s): The main expected result is to know the description of the use of Hypnotic Based Intervention for Non
Communicable Diseases sufferers. Regarding what techniques are used in Hypnotic Based Intervention, what types of diseases have been studied, what outcomes have been targeted, and what evidence can be used as an illustration that Hypnotic Based Intervention is effective.

Additional outcome(s): Additional expected results are findings about the factors that influence the success and failure of Hypnotic Based Intervention in patients suffering from Non Communicable Diseases.

Data management: Initially, the researchers selected the appropriate articles. After obtaining sufficient articles, the data synthesis process from the articles is carried out, preliminary synthesis is carried out using tabulations which include author, year of publication, location / country, target population, methods and main results. The form containing the main information was then reviewed by the research team and stake holders to ensure its relevance to the research and the validity of data extraction before continuing with the next investigation. The next step is to explore relationships, which is to link the findings from advanced searches through idea webbing and conceptual mapping. This is aimed at finding the relationship between research findings which can later be made a more complete and relevant concept to the research question. The data analysis was conducted qualitatively through the thematic narrative analysis method. The analysis categories will be arranged according to the main theme and answer the research questions, namely what types of Hypnotic Based Interventions are used for people with Non Communicable Diseases? What types of Non Communicable Diseases use Hypnotic Based Interventions? What are the outcomes studied to be targeted in the Hypnotic Based Therapy study in patients with Non Communicable Diseases? Is there evidence of the effectiveness of Hypnotic Based Interventions?

Quality assessment / Risk of bias analysis: To reduce the risk of bias, the data will be 
checked using RoB2 by the three reviewers. Disagreements will be resolved through voted. In addition, we use a clear way of reporting the findings of our research. We created tabs to map the findings to make it clearer based on the framework suggested by Arkey and O'Malley. Apart from getting articles relevant to the research question, we are also open to other findings that could be useful and have important implications for the future. The data obtained is then reviewed and arranged using tables, graphs and diagrams to make it easier for us to carry out thematic analysis.

Strategy of data synthesis: In conducting data synthesis, we use the methodology suggested by Arkey and O'Maller (2005). There are six steps that we take, including: 1) identifying research questions, 2) selecting appropriate research, 3) selecting studies, 4) mapping data, 5) conducting activities to compile, summarize and report the results of the study, 6) conduct discussions and hold meetings with stakeholders. To ensure that this scoping review research is well structured, we use the PRISMA ScR which consists of 20 essential reporting items and 2 optional items.

Subgroup analysis: If necessary and possible, the data obtained will be grouped on the type of intervention, the type of disease and the characteristics of the participants (for example, gender, age and education). The data obtained will be coded and then we process it.

Sensitivity analysis: We will study the text in detail from the selected articles and carry out a more careful filtering stage. By involving 3 reviewers who work independently, it will be able to increase the level of accuracy of the selected articles. We will perform detailed data extraction and report it systematically.

Language: English.

Country(ies) involved: Indonesia.
Keywords: Hypnosis, Hypnotherapy, NonCommunicable Diseases.

Contributions of each author:

Author 1 - Danang Baskoro - The first authors made plans, filtered articles and recorded data. Furthermore, the writer will extract and analyze the data. All planning activities were discussed with the third author.

Email: danangsetyobudibaskoro@gmail.com Author 2 - Leonardus Gandawijaya - The second author filters the initial data and ensures that the articles selected are relevant to the research theme.

Email: edwin.gandawijaya@gmail.com Author 3 - Kwartarini Yuniarti - The third writer filtered the baseline data, then made sure the articles selected match the research theme, and together with the first researcher to extract the data and analyze them.

Email: kwartarini_psy@ugm.ac.id 